ELECTRONIC RESEARCH ANNOUNCEMENTS OF THE AMERICAN MATHEMATICAL SOCIETY

Volume 3, Pages 55-62 (July 30, 1997)

S $1079-6762(97) 00024-3$

\title{
INERT ACTIONS ON PERIODIC POINTS
}

\author{
K. H. KIM, F. W. ROUSH, AND J. B. WAGONER
}

(Communicated by Douglas Lind)

\begin{abstract}
The action of inert automorphisms on finite sets of periodic points of mixing subshifts of finite type is characterized in terms of the sign-gyrationcompatibility condition. The main technique used is variable length coding combined with a "nonnegative algebraic K-theory" formulation of state splitting and merging. One application gives a counterexample to the Finite Order Generation Conjecture by producing examples of infinite order inert automorphisms of mixing subshifts of finite type which are not products of finite order automorphisms.
\end{abstract}

\section{INTRODUCTION, MAIN RESULTS, AND APPLICATIONS}

Subshifts of finite type $\left(X_{A}, \sigma_{A}\right)$ constructed from a nonnegative integral matrices $A$ appear in a number of areas ranging from smooth dynamical systems to coding and information theory. See $[\mathrm{LM}]$ for a comprehensive introduction to these model dynamical systems and to the field of symbolic dynamics in general. The automorphism group $A u t\left(\sigma_{A}\right)$ of $\left(X_{A}, \sigma_{A}\right)$ consists of those homeomorphisms of $X_{A}$ which commute with the basic shift homeomorphism $\sigma_{A}: X_{A} \longrightarrow X_{A}$. The first systematic study of $\operatorname{Aut}\left(\sigma_{A}\right)$ appeared in [He], and it has subsequently been studied in a number of papers. For example, see [BF], [BK1], [BLR], [KR1], [KRW1]. In general, $\operatorname{Aut}\left(\sigma_{A}\right)$ is a huge countable group. It contains copies of the direct sum of any countable collection of finite groups and is highly nonabelian. It contains a copy of the free abelian group on countably many generators. It contains copies of the fundamental groups of closed, 2-dimensional surfaces. It is residually finite, and therefore does not contain a divisible group. See [BLR]. The group $\operatorname{Aut}\left(\sigma_{A}\right)$ is interesting both in its own right and for its relation to other questions in symbolic dynamics. For example, present understanding of the fundamental classification problem for subshifts of finite type is closely related to $A u t\left(\sigma_{A}\right)$. See [B], [KRW1], [KR2], [W].

One way to study $\operatorname{Aut}\left(\sigma_{A}\right)$ is through representations of it to simpler groups, and there are currently two such representations which play a key role; namely, the periodic point and periodic orbit representations and the dimension group representation.

Received by the editors October 25, 1996.

1991 Mathematics Subject Classification. Primary 54H20, 57S99, 20 F99.

The first two authors were partially supported by NSF Grants DMS 8820201 and DMS 9405004. The last author was partially supported by NSF Grants DMS 8801333, DMS 9102959, and DMS 9322498 .

(C)1997 American Mathematical Society 
Let $P_{k}^{\bullet}=P_{k}^{\bullet}(A)$ denote the periodic points of period exactly $k$. Let $P_{k}=P_{k}(A)$ denote the disjoint union of $P_{l}^{\bullet}=P_{l}^{\bullet}(A)$ for $1 \leq l \leq k$. Let $A u t\left(\sigma_{A} \mid P_{k}^{\bullet}\right)$ denote the group of permutations of $P_{k}^{\bullet}$ which commute with $\sigma_{A}$. Similarly, let Aut $\left(\sigma_{A} \mid P_{k}\right)$ denote the group of permutations of $P_{k}$ which commute with $\sigma_{A}$. Then as in [BLR, Section 7] we have the periodic point representations

$$
\pi_{A, k}^{\bullet}: \operatorname{Aut}\left(\sigma_{A}\right) \longrightarrow \operatorname{Aut}\left(\sigma_{A} \mid P_{k}^{\bullet}\right)
$$

and

$$
\pi_{A, k}: \operatorname{Aut}\left(\sigma_{A}\right) \longrightarrow \operatorname{Aut}\left(\sigma_{A} \mid P_{k}\right) .
$$

Also discussed in [BLR, Section 7] are the closely related periodic orbit permutation representations

$$
\rho_{A, k, l}: \operatorname{Aut}\left(\sigma_{A}\right) \longrightarrow \bigoplus_{r=k}^{l} \operatorname{Perm}\left(\sigma_{A} \text { orbits of length } r\right)
$$

for $k \leq l$. Computing the images of $\pi_{A, k}$ and $\rho_{A, k, l}$ is a basic problem. If $\left(X_{A}, \sigma_{A}\right)$ is a primitive subshift of finite type, it is known from [BLR, 7.6] that $\rho_{A, k, l}$ is onto for $k$ sufficiently large. On the other hand, an example where $\left(X_{A}, \sigma_{A}\right)$ is primitive and $\pi_{A, 1}=\pi_{A, 1}^{\bullet}=\rho_{A, 1,1}$ is not onto is given in [KRW1, 4.3]. In Application 1 of our results below, we will show that in a number of cases the image of $\pi_{A, k}$ can be effectively determined for $k$ sufficiently large. This is relevant to the problem of classifying imbeddings of one shift into another of larger entropy as discussed in [BK2].

The periodic point representations gave rise to the Boyle-Krieger sign and gyration number homomorphisms

$$
O S_{A, k}: \operatorname{Aut}\left(\sigma_{A}\right) \longrightarrow \operatorname{Aut}\left(\sigma_{A} \mid P_{k}^{\bullet}\right) \longrightarrow Z / 2, \quad k \geq 1,
$$

and

$$
G Y_{A, k}: \operatorname{Aut}\left(\sigma_{A}\right) \longrightarrow \operatorname{Aut}\left(\sigma_{A} \mid P_{k}^{\bullet}\right) \longrightarrow Z / k, \quad k \geq 2,
$$

which were used in [BK1] to study involutions in $A u t\left(\sigma_{A}\right)$. The orbit sign number homomorphism $O S_{A, k}$ is simply the sign of the permutation induced on the orbits of length $k$ by an automorphism. The gyration number homomorphism $G Y_{A, k}$ is a measure of how automorphisms move orbits of length $k$ parallel to themselves.

The dimension group representation

$$
\delta_{A}: \operatorname{Aut}\left(\sigma_{A}\right) \longrightarrow \operatorname{Aut}\left(s_{A}\right)
$$

introduced by Krieger is essentially a matrix group representation which can be defined dynamically as in [BLR] or more algebraically as in [W]. The group $\operatorname{Aut}\left(s_{A}\right)$ of order preserving automorphisms of the dimension group is generally much simpler than $A u t\left(\sigma_{A}\right)$. It is often a torsion free, finitely generated abelian group. So typically, much of the complexity of $\operatorname{Aut}\left(\sigma_{A}\right)$ lies in the subgroup $\operatorname{Inert}\left(\sigma_{A}\right)$ of inert automorphisms which is defined to be the kernel of $\delta_{A}$. Application 2 below gives counterexamples to the FOG Conjecture about $\operatorname{Inert}\left(\sigma_{A}\right)$.

It turns out that the sign and gyration number homomorphisms are related to the dimension group representation through the so-called SGCC homomorphisms named after the sign-gyration-compatibility-condition which arose in the fundamental paper [BK1]. For $k \geq 2$, we have the SGCC homomorphisms

$$
S G C C_{A, k}: \operatorname{Aut}\left(\sigma_{A}\right) \longrightarrow \operatorname{Aut}\left(\sigma_{A} \mid P_{k}\right) \longrightarrow Z / k
$$


given in [KRW1], [W] by the formula

$$
S G C C_{A, k}=G Y_{A, k}+\sum_{i>0} O S_{A, k / 2^{i}}
$$

where we take $O S_{A, k / 2^{i}}=0$ whenever $k / 2^{i}$ is not an integer and where, for $k$ even, we consider $Z / 2$ as the subgroup $\{0, k / 2\}$ of $Z / k$. In particular, $S G C C_{A, k}=G Y_{A, k}$ whenever $k$ is odd, and

$$
S G C C_{A, 2}=G Y_{A, 2}+O S_{A, 1} .
$$

For simplicity of notation we shall usually just write $G Y_{k}=G Y_{A, k}, O S_{k}=O S_{A, k}$, and $S G C C_{k}=S G C C_{A, k}$. The relationship between the sign and gyration homomorphisms $O S_{k}$ and $G Y_{k}$ and the dimension group homomorphism $\delta_{A}$ is that $S G C C_{k}$ factors through $\delta_{A}$. See [KRW1]. We therefore know that

$$
S G C C_{k}(\alpha)=0 \quad \text { whenever } \alpha \text { lies in } \operatorname{Inert}\left(\sigma_{A}\right) \text {. }
$$

This paper announces the characterization of the action of inert automorphisms on the periodic points $P_{k}(A)$ for sufficiently large $k$, thereby computing the image of $\pi_{A, k}$ restricted to the inert subgroup $\operatorname{Inert}\left(\sigma_{A}\right)$. Details are available in the paper [KRW2]. The principal result is

Main Theorem. Assume that $A$ is primitive and $k \geq 2$ is a positive integer such that for every $n \geq k$ there is at least one periodic point of period exactly $n$. Let $\alpha$ be an element of Aut $\left(\sigma_{A} \mid P_{k}\right)$. Then there is an automorphism $\beta$ in $\operatorname{Inert}\left(\sigma_{A}\right)$ satisfying $\alpha=\beta \mid P_{k}$ iff $S G C C_{r}(\alpha)=0$ for every $2 \leq r \leq k$.

This is a corollary of the following three theorems.

Theorem A. Assume that $A$ is primitive and $2 \leq n \leq p$. Let $\alpha$ be an element of Aut $\left(\sigma_{A} \mid P_{n}^{\bullet}\right)$ such that $G Y_{n}(\alpha)=0$ and such that $\alpha$ induces the identity permutation on orbits of length $n$. Then there is an automorphism $\beta$ in Inert $\left(\sigma_{A}\right)$ satisfying

$$
\begin{aligned}
& \beta \mid P_{n}^{\bullet}=\alpha, \\
& \beta \mid P_{k}^{\bullet}=\text { Identity, for } 1 \leq k \leq p \text { and } k \neq n .
\end{aligned}
$$

Theorem B. Assume that $A$ is primitive and $1 \leq n \leq p$ are positive integers. Let $\alpha$ be an even permutation of the orbits of length $n$. Then there is an automorphism $\beta$ in Inert $\left(\sigma_{A}\right)$ satisfying

$$
\begin{aligned}
\alpha & =\text { the permutation induced on orbits of length } n \text { by } \beta, \\
G Y_{n}(\beta) & =0, \\
\beta \mid P_{k}^{\bullet} & =\text { Identity, for } 1 \leq k \leq p \text { and } k \neq n
\end{aligned}
$$

Theorem C. Assume that $A$ is primitive and $k \geq 2$ is a positive integer such that for every $n \geq k$ there is at least one periodic point of period exactly $n$. Let $\alpha$ be in Aut $\left(\sigma_{A} \mid P_{k}\right)$. Let $g y_{r}=G Y_{r}(\alpha)$ for $2 \leq r \leq k$ and let $o s_{r}=O S_{r}(\alpha)$ for $1 \leq r \leq k$. Assume that the sign-gyration-compatibility condition

$$
g y_{r}+\sum_{p>0} o s_{r / 2^{p}}=0
$$

holds for $2 \leq r \leq k$. Then there is an element $\beta$ in Inert $\left(\sigma_{A}\right)$ such that

$$
\begin{array}{lll}
G Y_{r}(\beta)=g y_{r} & \text { for } & 2 \leq r \leq k, \\
O S_{r}(\beta)=o s_{r} & \text { for } & 1 \leq r \leq k .
\end{array}
$$


Application 1. Computing the image of $\pi_{A, k}$ for $k$ large. Our three theorems above show how to find generators $\left\{\alpha_{1}, \ldots, \alpha_{p}\right\}$ for the subgroup

$$
\pi_{A, k}\left(\operatorname{Inert}\left(\sigma_{A}\right)\right) \subset \operatorname{Aut}\left(\sigma_{A} \mid P_{k}\right)
$$

for large enough $k$. If we can find a set of elements $\left\{\beta_{1}, \ldots, \beta_{q}\right\}$ in $\operatorname{Aut}\left(\sigma_{A}\right)$ so that the set $\left\{\delta_{A}\left(\beta_{1}\right), \ldots, \delta_{A}\left(\beta_{q}\right)\right\}$ generates the image of $A u t\left(\sigma_{A}\right)$ under the dimension group representation $\delta_{A}$, then $\left\{\alpha_{1}, \ldots, \alpha_{p}, \pi_{A, k}\left(\beta_{1}\right), \ldots, \pi_{A, k}\left(\beta_{q}\right)\right\}$ is a set of generators for the subgroup

$$
\pi_{A, k}\left(\operatorname{Aut}\left(\sigma_{A}\right)\right) \subset \operatorname{Aut}\left(\sigma_{A} \mid P_{k}\right) .
$$

In other words, if we can find automorphisms which generate the image of the dimension group representation, then we can find generators for the image of $\pi_{A, k}$ for $k$ large. For example, this applies to the full Bernoulli $n$-shift $\left(X_{n}, \sigma_{n}\right)$. A set of generators for the image of $\delta_{n}$ consists of the automorphisms

$$
\sigma_{r} \times I d_{n / r}: X_{r} \times X_{n / r} \longrightarrow X_{r} \times X_{n / r}
$$

where $r$ is a prime divisor of $n$.

Application 2. FOG Counterexamples. Boyle and Fiebig in $[\mathrm{BF}]$ characterized the action of products of finite order inert automorphisms on periodic points of mixing SFT's. Together with the theorems above, this produces many subshifts of finite type $\left(X_{A}, \sigma_{A}\right)$ having inert automorphisms which are not products of finite order inert automorphisms. One of the simplest examples is where

$$
A=\left(\begin{array}{llll}
1 & 1 & 0 & 0 \\
0 & 1 & 1 & 0 \\
0 & 0 & 1 & 1 \\
1 & 0 & 0 & 0
\end{array}\right) .
$$

According to Theorem B, the cyclic permutation (123) of the three fixed points is the restriction of an inert automorphism $\beta$ to the set of fixed points. On the other hand, since $\left(X_{A}, \sigma_{A}\right)$ has no points of period 2 or period $3,[\mathrm{BF}]$ says that any product of finite order inert automorphisms must be the identity on the fixed points. Incidentally, for this example it can be verified as in [BLR, 6.4] that the automorphism group $A u t\left(s_{A}\right)$ of the dimension group is a torsion free, finitely generated abelian group. Hence, every finite order element of $A u t\left(\sigma_{A}\right)$ is inert. So $\beta$ is not even a product of finite order automorphisms.

Heretofore, the big subgroups of infinite order elements in $\operatorname{Inert}\left(\sigma_{A}\right)$ were produced using very ingenious variations on the well-known "marker method" which gives rise to products of finite order elements. An interesting problem is to find natural representations of $\operatorname{Inert}\left(\sigma_{A}\right)$ into, say, a vector space or a torsion-free group which would detect the new infinite order inert automorphisms.

\section{POLYNOMIAL MATRICES}

The key technique we use is to present SFT's in terms graphs arising from square matrices with nonnegative polynomial entries (variable length coding) and to construct conjugacies between SFT's from row and column operations on these matrices (as in algebraic K-theory) but with certain natural positivity conditions brought in. A nonnegative polynomial matrix is one with entries in the set $t Z^{+}[t]$ of polynomials in the variable $t$ having nonnegative integer coefficients and zero constant term. If $A=(A(i, j))$ is a nonnegative polynomial matrix, construct the 
directed graph, or equivalently, the zero-one matrix $A^{\#}$ as follows: The indices $i$ and $j$ will be called the primary vertices of $A^{\#}$. Suppose

$$
A(i, j)=a_{1} t+a_{2} t^{2}+\cdots+a_{n} t^{n} .
$$

Corresponding to the term $a_{k} t^{k}$ in $A(i, j)$ draw $a_{k}$ simple paths of length $k$ from $i$ to $j$, each having $k$ edges and $k-1$ secondary vertices. By definition, we will let

$$
\left(X_{A}, \sigma_{A}\right)=\left(X_{A^{\#}}, \sigma_{A^{\#}}\right) .
$$

The usual SFT associated to a nonnegative integral matrix $A$ is obtained by the above construction from the nonnegative polynomial matrix $t A$.

Polynomial matrices turn out to be a very compact and efficacious way of representing SFT's and their automorphisms. For example, a special case of [BGMY, Theorem 1.7] shows that

$$
\operatorname{det}(I-A)=\operatorname{det}\left(I-t A^{\#}\right)
$$

So the Bowen-Lanford formula $[\mathrm{Sm}]$ for the zeta function yields

$$
\zeta_{A}(t)=\zeta_{A \#}(t)=\frac{1}{\operatorname{det}(I-A)} \text {. }
$$

There is a corresponding formula for dimension groups. Namely, there is an isomorphism of $Z\left[t, t^{-1}\right]$-modules

$$
\frac{Z\left[t, t^{-1}\right]^{n}}{\operatorname{Image}(I-A)} \cong \frac{Z\left[t, t^{-1}\right]^{n^{\#}}}{\operatorname{Image}\left(I-t A^{\#}\right)}
$$

This result has also been obtained by M. Boyle.

The polynomial matrix setting provides useful approximation lemmas. Suppose we have a sequence of nonnegative polynomial matrices

$$
A_{m}=B+t^{m} P_{m}
$$

where the entries $p(t)=p_{1} t+\cdots+p_{r} t^{r}$ of $P_{m}$ satisfy the conditions $r \leq D m$ and $p_{i} \leq C m^{e}$ for constants $e, C, D$ which are independent of $m$.

Entropy Limit Lemma. $\lim _{m \rightarrow \infty} h\left(A_{m}\right)=h(B)$.

Periodic Point Lemma. Let $A$ be a nonnegative polynomial matrix such that $A^{\#}$ is primitive. Assume

$$
\lim _{m \rightarrow \infty} h\left(A_{m}\right)=L<h(A) .
$$

Then there is an integer $K$ such that whenever $m \geq K$ we have

$$
\left|P_{q}^{\bullet}\left(A_{m}\right)\right| \leq\left|P_{q}^{\bullet}(A)\right| \quad \text { for } q \geq m
$$

We now discuss how the polynomial matrix setting can be used to produce strong shift equivalences and topological conjugacies between SFT's. If $a(t)=\sum_{r} a_{r} t^{r}$ and $b(t)=\sum_{r} b_{r} t^{r}$ are polynomials, we define $a \leq b$ iff $a_{r} \leq b_{r}$ for each $r$. Let $A$ be an $n \times n$ nonnegative polynomial matrix. Fix an entry $A(k, l)$ of $A$ where $k \neq l$, and let $b$ denote a polynomial in $t Z[t]$. Let $E_{k l}(b)$ denote the elementary matrix which is the identity on the diagonal and where the only nonzero entry off the diagonal is $b$ in the $k$ th row and $l$ th column. $E_{k l}(b)$ will be called a positive shear if $b \geq 0$. Let the matrix $B$ be defined by one of the following equations:

$$
\begin{aligned}
& I-B=E_{k l}(b)(I-A), \\
& I-B=(I-A) E_{k l}(b) .
\end{aligned}
$$


Positive Shear Lemma. Assume that $0 \leq b \leq A(k, l)$. Then $B$ is a nonnegative polynomial matrix and there are topological conjugacies

$$
\begin{aligned}
& R_{k l}(b):\left(X_{A^{\#}}, \sigma_{A^{\#}}\right) \longrightarrow\left(X_{B^{\#}}, \sigma_{B^{\#}}\right), \\
& C_{k l}(b):\left(X_{A^{\#}}, \sigma_{A^{\#}}\right) \longrightarrow\left(X_{B^{\#}}, \sigma_{B^{\#}}\right)
\end{aligned}
$$

corresponding respectively to the first and second equations above.

This lemma is proved using state splitting and merging [LM]. The relation to algebraic K-theory is that, roughly speaking, state splitting and merging corresponds to elementary row and column operations but with an extra positivity condition imposed. Conjugacies between subshifts of finite type are generated by the positive row and column type conjugacies $R_{k l}(b)$ and $C_{k l}(b)$ together with isomorphisms of graphs. This gives an algebraic formulation to the well-known classification theorem that conjugacies between SFT's are generated by state splitting and merging together with graph isomorphisms.

\section{IDEA OF PROOF}

The purpose of this section is to indicate the proof of a special case of Theorem B. Namely, assume that $\left(X_{A}, \sigma_{A}\right)$ is primitive and $P_{1}^{\bullet}(A)=\left\{p_{1}, \ldots, p_{r}\right\}$ for $r \geq 3$. Then the cyclic permutation (123) on fixed points may be realized by the action of an inert automorphism. The strategy is to construct certain inert automorphisms of model subshifts of finite type $\left(X_{C}, \sigma_{C}\right)$ corresponding to polynomial matrices $C=C_{m}$ such that

- $\sigma_{C}$ has exactly three fixed points and no other periodic points of period $q$ for $2 \leq q \leq m$.

- For $m$ large, $h(C)<h(A)$ and $\left|P_{s}^{\bullet}(C)\right| \leq\left|P_{s}^{\bullet}(A)\right|$ for $s \geq m$.

- $\left(X_{C}, \sigma_{C}\right)$ has an inert automorphism $\beta$ inducing (123) on fixed points.

Now let $X_{B}=X_{C} \cup\left\{p_{4}, \ldots, p_{r}\right\}$ and extend $\beta$ by letting it be the identity on $\left\{p_{4}, \ldots, p_{r}\right\}$. We then apply the Imbedding Theorem $[\mathrm{K}]$ and the Inert Extension Theorem [KR1] to imbed $\left(X_{B}, \sigma_{B}\right)$ into $\left(X_{A}, \sigma_{A}\right)$ and to extend $\beta$ to an inert automorphism of $\left(X_{A}, \sigma_{A}\right)$ as required.

To construct the model $\left(X_{C}, \sigma_{C}\right)$, we find $6 \times 6$ nonnegative polynomial matrices $C=C_{m}$ and $D=D_{m}$ such that

- $\sigma_{C}$ has exactly three fixed points $\left\{\mu_{1}, \mu_{2}, \mu_{3}\right\}$ and no other periodic points of period $q$ for $2 \leq q \leq m$.

- Let $\theta=(12)$. Then $C \theta=\theta C, \theta\left(\mu_{1}\right)=\mu_{2}$, and $\theta\left(\mu_{2}\right)=\mu_{1}$.

- $\delta_{C}(\theta)$ is in the center of $\operatorname{Aut}\left(s_{C}\right)$.

- $\sigma_{D}$ has exactly three fixed points $\left\{\nu_{1}, \nu_{2}, \nu_{3}\right\}$ and no other periodic points of period $q$ for $2 \leq q \leq m$.

- Let $\phi=(23)$. Then $D \phi=\phi D, \phi\left(\nu_{2}\right)=\nu_{3}$, and $\phi\left(\nu_{3}\right)=\nu_{2}$.

- There is a conjugacy $\gamma$ between $\left(X_{C}, \sigma_{C}\right)$ and $\left(X_{D}, \sigma_{D}\right)$ satisfying $\gamma\left(\mu_{i}\right)=\nu_{i}$ for $i=1,2,3$.

Let $\beta=\left[\theta, \gamma \phi \gamma^{-1}\right]$. Then $\beta$ is inert and is the cycle (123) on $\left\{\mu_{1}, \mu_{2}, \mu_{3}\right\}$.

Here is the algebraic formulation. We find $6 \times 6$ nonnegative polynomial matrices $C$ and $D$ so that

$$
D-I=S(C-I) T
$$

where $S$ and $T=S^{t}$ are certain products of positive shears which give rise to the desired conjugacy $\gamma . S$ is fixed in advance, and we show that $C$ can be chosen so 
that $C \theta=\theta C$ and so that the $D$ defined by (1) is nonnegative and $D \phi=\phi D$. This involves solving a set of homogeneous linear equations satisfied by the unknown $X=C-I$ with coefficients which are products of the entries of $S$. To control the number of low order periodic points, there is a congruence condition which must be satisfied by $X$ modulo $t^{m}$. We also require that $X$ satisfies a certain congruence condition modulo $\Delta^{2}$, where $\Delta=t-1-t^{m}$, as well as an additional nonhomogeneous linear equation

$$
X_{11}-X_{12}=\Delta .
$$

This will insure that there is a decomposition of the dimension group

$$
\operatorname{Coker}(I-C) \otimes Q=K_{-} \oplus K_{+}
$$

as a $Q\left[t, t^{-1}\right]$-module where $K_{-}$and $K_{+}$are the -1 and +1 eigenspaces of $\theta$ and where $K_{-}$and $K_{+}$have relatively prime annihilators. So any $Q\left[t, t^{-1}\right]$-module isomorphism of Coker $(I-C) \otimes Q$ must preserve (3), and consequently $\delta_{C}(\theta)$ will be in the center of $A u t\left(s_{C}\right)$.

The proof of Theorem A is very similar to that of Theorem B. In both cases, the key step is to construct inert automorphisms of model SFT's with low entropy and good control over the growth of periodic points. This is done by fixing a priori certain products of positive shears $S$ and $T=S^{t}$, and then showing how to choose $C$ so that the matrix $D$ defined by

$$
D-I=S(C-I) T
$$

satisfies a symmetry condition. The proof of Theorem $\mathrm{C}$ is somewhat different. We first construct a priori nonnegative polynomial matrix models $C_{1}, D_{1}$ and products of shears $S_{1}, T_{1}$ so that

$$
D_{1}-I=S_{1}\left(C_{1}-I\right) T_{1}+Y\left(t^{m} \Delta^{2}\right)^{r}
$$

for a certain polynomial $\Delta$ which is relatively prime to $t^{m}$. Then in several steps we enlarge $C_{1}$ to another matrix $C$ satisfing an involution symmetry condition and an appropriate eigenspace determinant condition, and we multiply $S_{1}$ and $T_{1}$ on the left and right respectively by additional products of shears depending on the error term $Y\left(t^{m} \Delta^{2}\right)^{r}$ to obtain new products of shears $S$ and $T$ so that the matrix $D$ defined by

$$
D-I=S(C-I) T
$$

satisfies another involution symmetry condition and the same eigenspace determinant condition and so that $S$ and $T$ satisfy certain positive shear conditions.

\section{ACKNOWLEDGEMENT}

We would like to thank Mike Boyle and Doug Lind for conversations about this material.

\section{REFERENCES}

[B] M. Boyle, The stochastic shift equivalence conjecture is false, Contemporary Mathematics, Vol. 135, 1992, 107-110. MR 93i:28013

[BF] M. Boyle and U. Fiebig, The action of inert finite order automorphisms on finite subsystems, ETDS 11 (1991), 413-425. MR 92g:58029

[BGMY] L. Block, J. Guckenheimer, M. Misiurewicz, and L.-S. Young, Periodic points and entropy of one dimensional maps, Springer Verlag Lecture Notes in Mathematics No. 819, 1980, pp. 18-34. MR 82j:58097 
[BK1] M. Boyle and W. Krieger, Periodic points and automorphisms of the shift, TAMS 302 (1987), 125-149. MR 88g:54065

[BK2] M. Boyle and W. Krieger, Automorphisms and subsystems of the shift, Jour. Reine Angew. Math. 437 (1993), 13-28. MR 95b:54051

[BLR] M. Boyle, D. Lind, and D. Rudolph, The automorphism group of a shift of finite type, TAMS 306 (1988), 71-114. MR 89m:54051

[He] G. Hedlund, Endomorphisms and automorphisms of the shift dynamical systems, Math. Systems Theory 3 (1969), 320-375. MR 41:4510

[K] W. Krieger, On subsystems of topological Markov chains, ETDS 2 (1982), 195-202. MR 85b:28020

[KR1] K. H. Kim and F. W. Roush, On the structure of inert automorphisms of subshifts, Pure Math. Appl. Ser. B 2 (1991), 3-22. MR 94c:58056

[KR2] K. H. Kim and F. W. Roush, Williams' conjecture is false for reducible subshifts, JAMS 5, no. 1 (1992), 213-215. MR 92j:54055

[KRW1] K. H. Kim, F. W. Roush, and J. B. Wagoner, Automorphisms of the dimension group and gyration numbers, JAMS, 5, no.1 (1992), 191-212. MR 93h:54026

[KRW2] K. H. Kim, F. W. Roush, and J. B. Wagoner, Characterization of inert actions on periodic points, preprint, UC Berkeley, July 1996.

[LM] D. Lind and B. Marcus, An introduction to symbolic dynamics and coding, Cambridge University Press, 1995. MR 97a:58050

[Sm] S. Smale, Differentiable dynamical systems, BAMS 73, no. 6 (1967), 747-847. MR $\mathbf{3 7 : 3 5 9 8}$

[W] J. B. Wagoner, Classification of subshifts of finite type revisited, Contemporary Mathematics, Vol. 135, 1992, 423-436. MR 93m:20004

Department of Mathematics, Alabama State University, Montgomery, Alabama 36101

E-mail address: kkim@asu.alasu.edu

Department of Mathematics, Alabama State University, Montgomery, Alabama 36101

E-mail address: kkim@asu.alasu.edu

Department of Mathematics, UCB, Berkeley, California 94720

E-mail address: wagoner@math.berkeley.edu 